\title{
Optical damage thresholds of microstructures made by laser 3D nanolithography
}

\author{
Agnè Butkutè ${ }^{1}$, Laurynas čekanavičius ${ }^{1}$, Gabrielius Rimšelis ${ }^{1}$, Darius Gailevičius ${ }^{1}$, \\ Vygantas Mizeikis $^{2}$, Andrius Melninkaitis ${ }^{1,3}$, Tommaso Baldacchini ${ }^{4,5}$, \\ Linas Jonušauskas ${ }^{1,6}$, and Mangirdas Malinauskas ${ }^{1}$
}

\author{
${ }^{1}$ Laser Research Center, Vilnius University, Sauletekis Ave. 10, Vilnius LT-10223, Lithuania \\ ${ }^{2}$ Research Institute of Electronics, Shizuoka University, 3-5-1 Johoku, Naka-ku, Hamamatsu 432-8561, Japan \\ ${ }^{3}$ Lidaris Ltd., Saulètekio Al., 10, Vilnius LT-10223, Lithuania \\ ${ }^{4}$ Schmid College of Science and Technology, Chapman University, One University Drive, Orange, CA 92866, USA \\ ${ }^{5}$ Department of Chemistry, University of California, Irvine, California 92697, USA \\ ${ }^{6}$ Femtika Ltd., Sauletekio Ave. 15, Vilnius LT-10224, Lithuania \\ *Corresponding authors: linas@femtika.It,mangirdas.malinauskas@ff.vu.It
}

\begin{abstract}
Direct laser writing based on non-linear 3D nanolithography (also known as 3D laser lithography, 3DLL) is a powerful technology to manufacture polymeric micro-optical components. However, practical applications of these elements are limited due to the lack of knowledge of their optical resilience and durability. In this work, we employ 3DLL for the fabrication of bulk (i.e. fully filled) and woodpile structures out of different photopolymers. We then characterize them using S-on-1 laser induced damage threshold (LIDT) measurements. In this way, quantitative data of LIDT values can be collected. Furthermore, this method permits to gather damage morphologies. The results presented in this work demonstrate that LIDT values depend on the material and the geometry of the structure. Bulk non-photosensitized hybrid organic-inorganic photopolymer SZ2080 structures are found to be the most resilient with a damage threshold being of $169 \pm 15 \mathrm{~mJ} / \mathrm{cm}^{2}$.
\end{abstract}

\section{Introduction}

3D laser lithography (3DLL) is a rapidly developing technique that allows the fabrication of micro and nano-scale 3D objects. It is used in a multitude of fields, and it has the potential to be implemented in several industrial processes ?. One of the key areas of 3DLL is the production of micro-optics. It has been demonstrated that such structures can be used for spatial light modulation? and imaging purposes? ${ }^{?}$. They can also be integrated on functional substrates such as fiber tips ${ }^{?}$ or imaging devices? ${ }^{?}$. However, in order to implement these components in applications where high light intensities $\left(>\mathrm{GW} / \mathrm{cm}^{2}\right)$ are used, such as laser material processing and laser scanning microscopy, it is important to evaluate their laser induced damage threshold (LIDT).. Some preliminary studies have been carried out in this direction already, but these either used thin films, which are structurally very different to 3DLL made objects ${ }^{?}$, or gave only qualitatively guidelines ${ }^{?}$. This work is aimed at determining LIDT values for microstructures made specifically by 3DLL. For this purpose, 3DLL microstructures fabricated using different polymers and writing schemes are tested to uncover trends in LIDT values that depend on both the material and internal porosity of the microstructures.

\section{Materials and methods}

Structuring and LIDT evaluation are performed by using laser Nanopolymerization setup of Vilnius University Laser Research

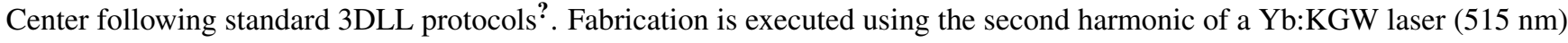
with pulse duration of $300 \mathrm{fs}$ and $200 \mathrm{kHz}$ repetition rate $(f)$. To focus the laser beam, several objectives are used depending on the structure to be fabricated. For the formation of filled microstructures, a 20x 0.45 numerical aperture (NA) objective is used that allows the fastest structuring rate? ${ }^{?}$. Woodpile microstructures require the use of a $63 \mathrm{x} 1.4$ NA objective since, in this case, it is necessary to achieve sub- $\mu \mathrm{m}$ feature size. The woodpiles were defined by their transverse (axy) and longitudinal (az) periods as well as their line width in horizontal $(d x y)$ and vertical $(d z)$ directions. To evaluate LIDT values for each investigated polymer, $10 \times 10$ arrays of either filled or woodpile microstructures are produced. The dimensions of the microstructures are $100 \times 100 \times 30 \mu \mathrm{m}^{3}$. The microstructures are separated by gaps of $150 \mu \mathrm{m}$. Exact structuring parameters can be found in 
previous works by our group?,? Before performing LIDT tests, all microstructure arrays are investigated by scanning electron microscopy (SEM) to ensure they are all made without any defects which could negatively affect LIDT measurements.

LIDT tests are performed by using a guidance of so called ISO S-on-1 method with a fs-laser pulse trains?. This method delivers statistically determined LIDT values, as well as damage morphologies. A representation of a typical LIDT experiment is shown in Fig. 1. 3DLL microstructures are exposed to the focused laser beam one by one. The focal length of the lens used is $f_{l}=10 \mathrm{~cm}$, resulting in a beam waist of $2 \omega_{0}=20 \mu \mathrm{m}$ at $1 / \mathrm{e}^{2}$ level. The fundamental harmonic $(1030 \mathrm{~nm}) \mathrm{of}$ the $\mathrm{Yb}: \mathrm{KGW}$ laser is used with $300 \mathrm{fs}$ pulse duration and $100 \mathrm{kHz}$ repetition rate. The experiment is monitored in real time by a CMOS camera integrated into the experimental setup. Each row of the $10 \mathrm{x} 10$ microstructure arrays is exposed to a different laser average power $P$. The exposure time is kept constant at one minute (6 million laser pulses for each exposure site). After all microstructures are exposed to the laser light, the sample is examined by SEM and optical microscopy to evaluate which microstructures are damaged and which ones are not. Criteria of damage was any kind of observable modification. It was observed at relatively similar levels for both SEM and optical microscope. Probability ( $p$ ) to damage a microstructure at a particular fluence $\left(F=P / 2 \omega_{0} f\right)$ is evaluated next. It is extrapolated by performing a linear approximation of the dynamic range in which $0 \%<p<100 \%$. The last and first $F$ corresponding to $p=0 \%$ and $p=100 \%$ are also included. As by S-on- 1 ISO standard, the $F(p)$ is approximated to a linear function. The intersection of the slope at $p=0 \%$ is considered the LIDT value $\left(F_{t}\right)$ of investigated structure. The error associated with measured LIDT values is set equal to the single measurement step employed in the applied $F$.

\section{Results and discussion}

We begin our work by investigating how LIDT values depend on the material. For this purpose, we prepared an array of 3DLL made rectangular bulks. Popular photopolymers are investigated to represent lithographic materials. Namely they are a resin used in typical stereolithographic processes (PR48 from Autodesk), the popular epoxy-based resin SU8, and two hybrid organic-inorganic resins (OrmoClear and SZ2080) ${ }^{\text {? }}$. SZ2080 was used with either of the two following photoinitiators, 4,4'-bis(dimethylamino)benzophenone or 2-benzyl-2-dimethylamino-1-(4 morpholino phenyl)-butanone-1. We will refer to these two photoinitiators using the abbreviations BIS and IRG, respectively. Furthermore, SZ2080 was used as is without a photoinitiator. In the following, these photoinitiators will be referred to by using abbreviations BIS and IRG, respectively. Experimental results of LIDT tests are shown in Fig. 2. The highly photoactive PR48 has the lowest LIDT value of $F_{t}=4 \pm 3 \mathrm{~mJ} / \mathrm{cm}^{2}$. The second lowest LIDT value belongs to SZ2080 with $1 \% \mathrm{wt}$. BIS: $F_{t}=17 \pm 9 \mathrm{~mJ} / \mathrm{cm}^{2}$. This result can partly be explained by the relatively long wavelength absorption of BIS that reaches into the visible part of the spectrum $\left(\sim 520 \mathrm{~nm}^{?}\right)$. The LIDT value for SU8 is $F_{t}=36 \pm 6 \mathrm{~mJ} / \mathrm{cm}^{2}$ (absorption peak $-360 \mathrm{~nm}$ ). SZ2080 with $1 \%$ wt. IRG and OrmoClear followed with $F_{t}=42 \pm 15 \mathrm{~mJ} / \mathrm{cm}^{2}$ and $F_{t}=51 \pm 15 \mathrm{~mJ} / \mathrm{cm}^{2}$, respectively. This can be expected as both of these materials are glassy hybrids with absorption in the UV $\left(\sim 390 \mathrm{~nm}^{?, ?}\right.$ and $\sim 375 \mathrm{~nm}^{?}$ respectively). Finally, SZ2080 with no photoinitiator outperformed all other materials with a LIDT value of $F_{t}=169 \pm 15 \mathrm{~mJ} / \mathrm{cm}^{2}$. The absorption spectrum of non-photosensitized SZ2080starts as low as $340 \mathrm{~nm}^{\text {?,? }}$ ). In all of these cases absorption was measured in materials as if they were prepared for fabrication. With $F_{t}$ plotted as function of absorption we can see a clear correlation. This can be tied with lower order of nonlinearity for tested $1030 \mathrm{~nm}$ radiation as absorption peak moves to longer wavelengths. Furthermore, the tendency of hybrids being more resilient that organic polymers at same absorption values is also visible.

Besides absorption, another material characteristic that can influence damage mechanisms is its homogeneity. The linear regression shown in Fig. 2 is an indication of how homogeneous a fabricated microstructure is. All three worst performing materials have damage dynamic ranges that are relatively narrow, going from $p=0 \%$ to $p=100 \%$ in less than $50 \mathrm{~mJ} / \mathrm{cm}^{2}$. On the contrary, SZ2080 with $1 \%$ wt. IRG and OrmoClear have damage dynamic ranges that cover $100 \mathrm{~mJ} / \mathrm{cm}^{2}$. Therefore, somewhat inhomogeneous (in terms of LIDT) nature of material at nano-scale can be considered. In such case different nano-constitutes/impurieties of the material have varying LIDT $^{?}$. Beginning of dynamic damage range starts when constitute having lower LIDT starts being damaged and reaches full 100\% when even the more resilient part is being fully affected every time. In this case the source of such inhomogeneity might be the photoinitiators used because, with pure material, dynamic range is again reduced to around $50 \mathrm{~mJ} / \mathrm{cm}^{2}$. Interestingly, the definite damage region $(p=100 \%)$ for both pure and IRG photosensitized SZ2080 is basically the same, starting at roughly $\sim 200 \mathrm{~mJ} / \mathrm{cm}^{2}$. Due to this we can consider photoinitiator to form small conglomerates of lower $F_{t}$ that reduce the overall LIDT value.

Interpretation of the LIDT values for the filled 3DLL microstructures is relatively straightforward since these objects do not induce any intricate light-matter interactions. However, complex 3DLL microstructures such as woodpiles can influence light in a multitude of ways? Thus, predicting and interpreting LIDT values in this scenario is a non-trivial task. One must consider the fill ratio of such microstructures as well as the ratios between $d x y$, axy and $a z$ [Fig. 1]. The latter ones influence the volume/surface area of the woodpiles and their photonic properties. Therefore, we study the effect of aforementioned parameters when performing LIDT tests of woodpiles microstructures. For these tests, we narrowed down the materials to pure SZ2080 and SZ2080 with 1\% wt. IRG. These materials have been previously fully investigated by 3DLL?, making parameters 
optimization an easier task .

During woodpile testing $a x y=1.13 \mu \mathrm{m}$ and $a z=0.8 \mu \mathrm{m}$ were kept constant in order to make sure that the structures are transparent for $1030 \mathrm{~nm}$ radiation?. Only $d x y$ was varied thus changing fill ratio and Surface area $S$ and internal volume $V$ of polymerized lines. The values of fill ratio, $V$ and $S$ were calculated assuming lines to be elipsoids? ${ }^{?}$. Material in this test was pure SZ2080. It is known that the surface defects are one of the primary reasons for optical damage in thin films? ${ }^{\text {. Thus, }}$ the goal here was to check if relatively high $S$ and small $V$ would lead to lower LIDT. The calculation was done assuming the lines in woodpile to have elliptical profile and calculating the internal volume and surface area of such rod. Having $V$ allows to calculate fill factor $(F F)$, showing how much of volume is taken up by lines in comparison to full same sized bulk. Indeed, with a lot of volume in the structure taken up by air, the thermal diffusion should be minimized. Thus, if LIDT would depend on $F F$ it would point out to the strong thermal nature of the effect. Thus, LIDT values of woodpiles depend on changes in $S / V$ and $F F$ are shown in Fig. 3 (a). No clear dependency can be deduced, thus showing minimal LIDT dependence on woodpiles $F F$ and $S / V$ ratio with maximal LIDT value $F_{t}=139.5 \pm 15 \mathrm{~mJ} / \mathrm{cm}^{2}$ at axy $=400 \mathrm{~nm}$.

Due to the woodpile geometry, strength of electrical field $E$ inside of such structure can have some internal peaks. Mathematical modeling was performed in order to visualize it. Standard FDTD modeling procedure described in the literature was used? ${ }^{?}$. Due to peculiarities of mathematical model used only $d x y=500 \mathrm{~nm}, 450 \mathrm{~nm}$ and $400 \mathrm{~nm}$ could be calculated. The modeling shows that changes in axy can increase $E^{2}$ value by $\sim 23$ time comparing the lowest and the highest value [Fig. 3 (b)] at $d x y=400 \mathrm{~nm}$ and $d x y=500 \mathrm{~nm}$ respectively. These peaks are located inside of the woodpile lines, which denotes possible influence to LIDT. Geometries with such a significant increase in $E^{2}$ also proved to have $\sim 27.3 \%$ lower LIDT. Therefore relation between woodpile's photonic properties and its LIDT is evident. Therefore, a care should be taken when using 3D woodpile type structures in high light intensities even if they should be transparent to usable wavelength, as internal distribution of $E^{2}$ might yield some specific peaks that could consequently damage the structure.

Woodpile testing protocol was repeated with photosensitized woodpiles. The highest acquired LIDT was $F_{t}=105 \pm 7.5 \mathrm{~mJ} / \mathrm{cm}^{2}$. As expected, it was lower than LIDT of the most resilient non-photosensitized SZ2080 woodpile by $\sim 24.8 \%$. On the other hand, it exceeded the value acquired with the same photopolymer composition in bulk configuration. This shows that smaller $V$ of absorbing woodpile outweights potential phonic $E^{2}$ confinement. Nevertheless, this was not noticed in less absorbing pure material. At the same time LIDT difference is a lot smaller between woodpiles that between bulk objects $(\sim 77.4 \%$ difference) meaning that geometry is more important than material absorption in the case of woodpiles.

Finally, it is instructive to outline some characteristic structural features of laser-induced damage in some of the investigated materials detail. Fig. 4 shows SEM images of damage sites in PR48 and pure SZ2080, which correspond to materials that exhibited extreme values of LIDT. As can be seen from Fig. 4 (a), optical damage in PR48 does not result in formation of crater characteristic for dielectric breakdown countrary to pure SZ2080 (Fig. 4 (b)). It is likely the result of relatively low crosslinking degree of PR48. In comparison, structural damage in SZ2080 shown in Fig. 4 (b) exhibits all typical features of Coulomb explosion-driven dielectric breakdown? Here, a void at the center of the focus, surrounded by a densified region, can be clearly seen. It can be tied to phase transfer from hybrid polymer to ceramics under sever heat ${ }^{\text {}}$. Hence, we can conclude that low damage threshold in PR48 can be mainly attributed to its soft, possibly partially polymerized state rather than spectral position of the linear absorption edge, $\lambda_{a} \approx 430 \mathrm{~nm}$, in the standard photoinitiator Esstech TPO+ used to photosensitize PR48? Examples of optical damage in 3D structured pure SZ2080 for two different laser $F$ are shown in Fig. 4 (c) and (d). At lower $F$ (Fig. 4 (c)), a crater with well-defined edges, and without signs of melting or heat-affected zone is seen at the center of focus. Closer inspection of areas surrounding the crater reveals slight reduction of the woodpile lattice period, which becomes even more evident at high $F$ (Fig. 4 (d)). It indicates Coulomb explosion as the dominant mechanism of dielectric breakdown in periodic structures in SZ2080.

\section{Conclusions}

In conclusion, this work provided new insights into the dynamics of optical damage of 3DLL made structures. All the acquired quantitative LIDT values plotted in Fig. 2 showed that LIDT severely depends on the material (organic vs hybrid) and its composition (photosensitized $v s$ without PI), with hybrid organic-inorganic photopolymers clearly outperforming the rest of the materials tested. The significance of photoinitiator can be explained by increased absorption at longer wavelengths as well as possible introduction of nanoinhomogeneity. Additionally, testing of woodpiles uncovered LIDT peculiarities of 3D nano-lattices. It was shown that for photonically transparent woodpiles neither $S / V$ ratio nor $F F$ have noticeable influence to the LIDT. Conversely, photonic properties of woodpile resulting in internal redistribution of $E^{2}$ have potential influence to LIDT due to the possible changes in $E^{2}$ by up to 23 times. Field amplification offset the absorption dominating in bulk case. As a result photosensitized woodpiles outperform bulk counterparts, while pure SZ2080 woodpiles perform worse than non-photosensitized bulk. Also, the difference between woodpile LIDT values is smaller than between bulks. In result, the best performing 3DLL made structure in this study was bulk made out of SZ2080 without PI. It follows the tendency showed in previous qualitative study? and has LIDT of $F_{t}=169 \pm 15 \mathrm{~mJ} / \mathrm{cm}^{2}$. Such value is around 4 times lower to measured in thin film 
case $\left(F_{t}=570 \pm 80 \mathrm{~mJ} / \mathrm{cm}^{2}\right)^{?}$ and around on order of magnitude smaller than that of fused silica $\left(F_{t}=3.21 \pm 0.11 \mathrm{~J} / \mathrm{cm}^{2}\right)^{?}$.

Funding. NATO (No. SPS-985048) Grant and European Commission's Seventh Framework Programme Laserlab-Europe IV JRA scheme BIOAPP (No. EC-GA 654148) for the financial support.

Disclosures. The authors declare no conflicts of interest.

\section{References}

1. Malinauskas, M. et al. Ultrafast laser processing of materials: from science to industry. Light. Sci. Appl. 5, e16133, DOI: 10.1038/lsa.2016.133 (2016).

2. Maigyte, L. et al. Flat lensing in the visible frequency range by woodpile photonic crystals. Opt. Lett. 38, 2376-2378 (2013).

3. Gissibl, T., Thiele, S., Herkommer, A. \& Giessen, H. Two-photon direct laser writing of ultracompact multi-lens objectives. Nat. Photonics 10, 554-560, DOI: 10.1038/nphoton.2016.121 (2016).

4. Williams, H. E., Freppon, D. J., Kuebler, S. M., Rumpf, R. C. \& Melino, M. A. Fabrication of three-dimensional microphotonic structures on the tip of optical fibers using SU-8. Opt. Express 19, 22910-22922, DOI: 10.1364/oe.19.022910 (2011).

5. Thiele, S., Arzenbacher, K., Gissibl, T., Giessen, H. \& Herkommer, A. M. 3D-printed eagle eye: Compound microlens system for foveated imaging. Sci. Adv. 3, e1602655 (2017).

6. Žukauskas, A. et al. Characterization of photopolymers used in laser 3D micro/nanolithography by means of laser-induced damage threshold (LIDT). Opt. Mater. Express 4, 1601-1616, DOI: 10.1364/ome.4.001601 (2014).

7. Jonušauskas, L. et al. Optically clear and resilient free-form $\mu$-optics 3D-printed via ultrafast laser lithography. Materials 10, 12, DOI: 10.3390/ma10010012 (2017).

8. Jonušauskas, L. et al. Mesoscale laser 3D printing. Opt. Express 27, 15205-15221, DOI: 10.20944/preprints201810.0384.v1 (2019).

9. ISO21254-2:2011. Test methods for laser-induced damahe threshold-Part 2: Threshold determination (2011).

10. Ovsianikov, A. et al. Ultra-low shrinkage hybrid photosensitive material for two-photon polymerization microfabrication. Acs Nano 2, 2257-2262 (2008).

11. Malinauskas, M., Žukauskas, A., Bičkauskaitė, G., Gadonas, R. \& Juodkazis, S. Mechanisms of three-dimensional structuring of photo-polymers by tightly focussed femtosecond laser pulses. Opt. Express 18, 10209 (2010).

12. Microresist. UV-Curable Hybrid Polymers for Micro Optical Components (2015).

13. Batavičiutè, G., Grigas, P., Smalakys, L. \& Melninkaitis, A. Revision of laser-induced damage threshold evaluation from damage probability data. Rev. Sci. Instrum. 84, 045108, DOI: 10.1063/1.4801955 (2013).

14. Batavičiūtè, G., Ščiuka, M. \& Melninkaitis, A. Direct comparison of defect ensembles extracted from damage probability and raster scan measurements. J. Appl. Phys. 118, 105306, DOI: 10.1063/1.4929963 (2015).

15. Mizeikis, V., Purlys, V., Buividas, R. \& Juodkazis, S. Realization of structural color by direct laser write technique in photoresist. J. Laser. Micro. Nanoeng. 9, 42-45 (2014).

16. Juodkazis, S., Misawa, H., Hashimoto, T., Gamaly, E. G. \& Luther-Davies, B. Laser-induced microexplosion confined in a bulk of silica: Formation of nanovoids. Appl. Phys. Lett. 88, 201909, DOI: 10.1063/1.2204847 (2006).

17. Gailevičius, D. et al. Additive-manufacturing of 3D glass-ceramics down to nanoscale resolution. Nanoscale Horiz. 4, 647-651, DOI: 10.1039/c8nh00293b (2019).

18. Bennett, J. Measuring UV curing parameters of commercial photopolymers used in additive manufacturing. Addit. Manuf. 18, 203-212, DOI: 10.1016/j.addma.2017.10.009 (2017).

19. Gallais, L. \& Commandré, M. Laser-induced damage thresholds of bulk and coating optical materials at $1030 \mathrm{~nm} 500 \mathrm{fs}$. Appl. Opt. 53, A186-A196, DOI: 10.1364/ao.53.00a186 (2013). 


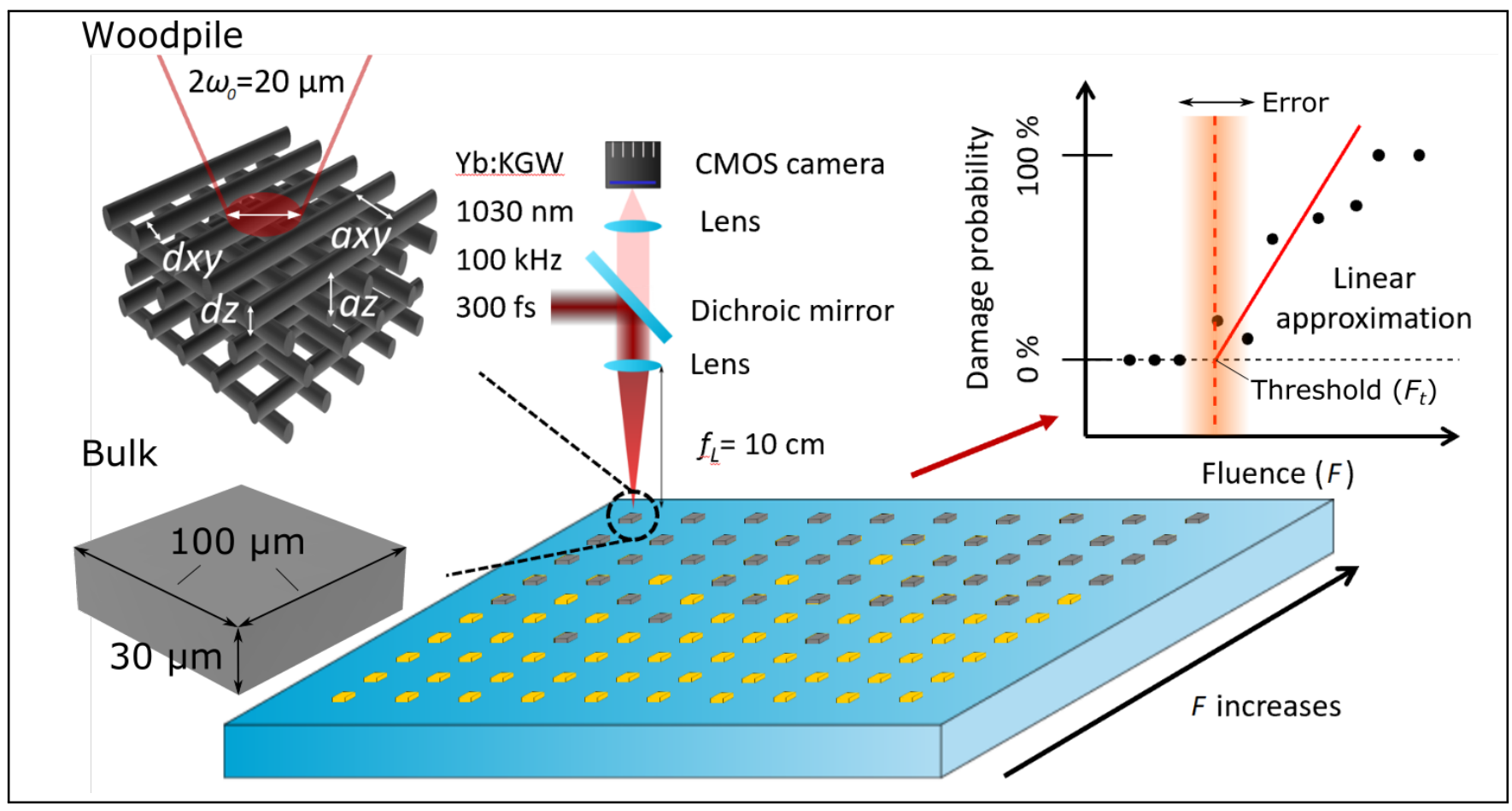

Figure 1. Schematics of LIDT measurement. A 10 x 10 array of either bulks or woodpiles is exposed to $1030 \mathrm{~nm} 100 \mathrm{kHz}$ $300 \mathrm{fs}$ laser radiation. Both bulks and woodpiles were $100 \mu \mathrm{m}$ x $100 \mu \mathrm{m}$ x $30 \mu \mathrm{m}$ in size, with woodpile being arranged in fcc geometry. Each woodpile was also characterized by line width $d x y$, distance between them $a x y$ and period $a z$. $F$ was changed from line to line, from no damage to all structures being damaged. This allowed to plot damage probability as a function of $F$. Linear approximation was then used to determine $F_{t}$. Error is considered to be single $F$ step. 


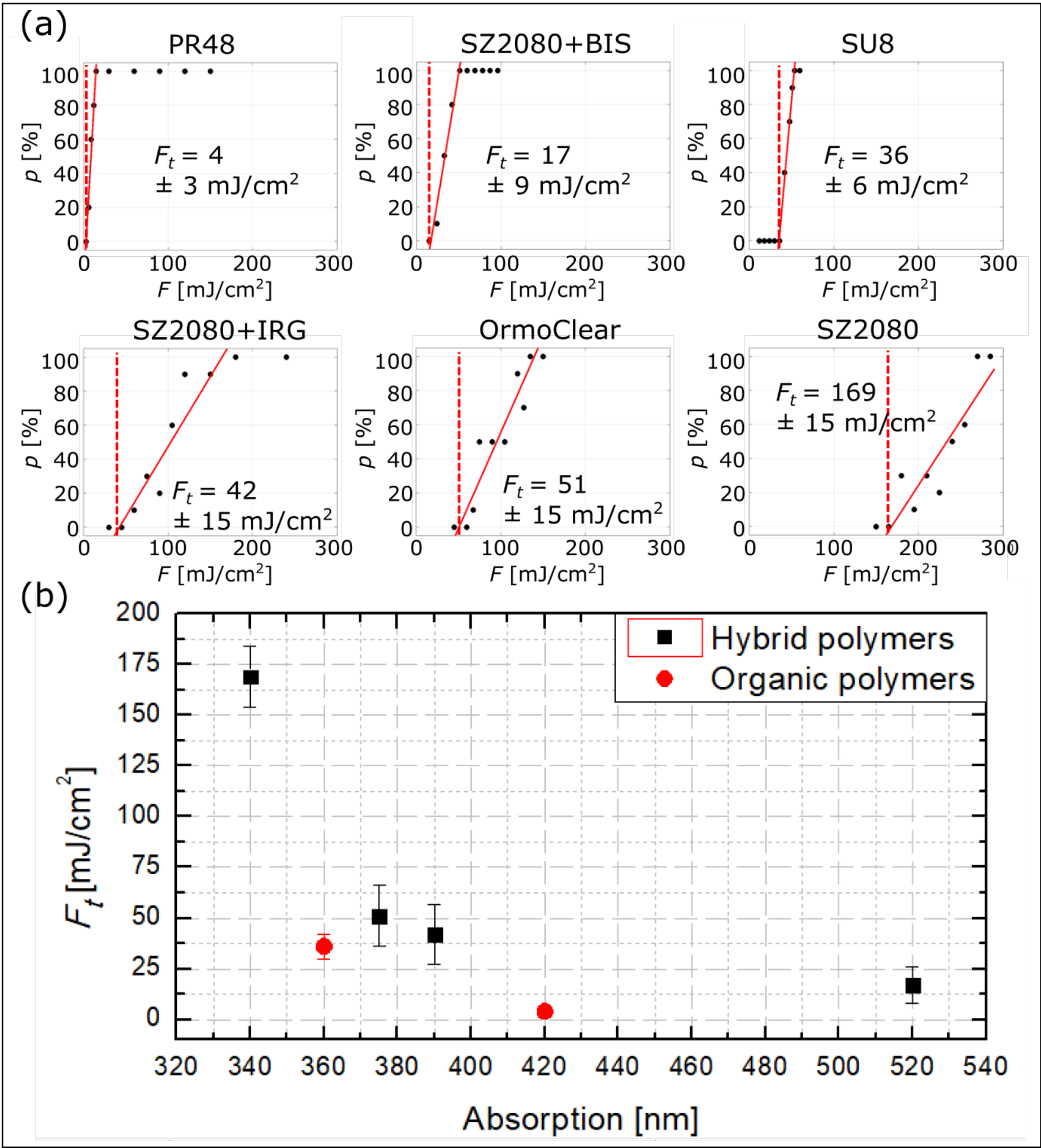

Figure 2. (a) LIDT values of bulks for all tested materials. Solid red lines represent linear approximation, red dashed lines the threshold $F$. SZ2080 with no PI is the most resilient with $F_{t}=161.9 \pm 12.5 \mathrm{~mJ} / \mathrm{cm}^{2}$. (b) $F_{t}$ depends on the absorption of the material. Hybrid polymers are also more resilient at the same absorption than pure organics. 


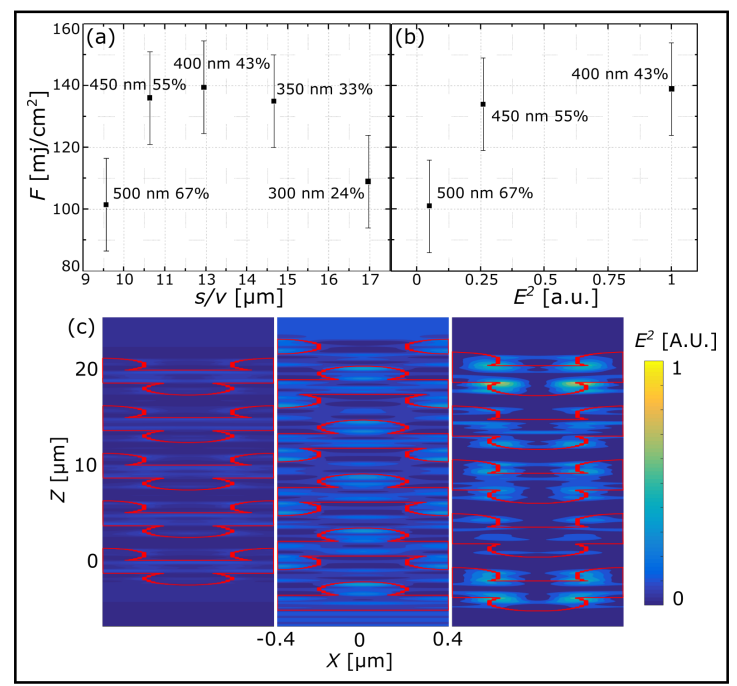

Figure 3. LIDT dependency on $S / V$ ratio (a) $E^{2}$ (b) and $F F$ (written near each measured point alongside $d x y$ value). (c) result of $E^{2}$ modeling inside of woodpiles with the three thickest $d x y$ values. Regions enclosed by the red line show the cut of internal features of woodpile. Difference between the highest (at $d x y=500 \mathrm{~nm}$ ) and lowest (at $d x y=400 \mathrm{~nm}$ ) $E^{2}$ is $\sim 23$ times. This follows tendency of increase in LIDT between these two cases by $\sim 27.3 \%$ showing that optical resiliency depends on photonic properties of woodpile.

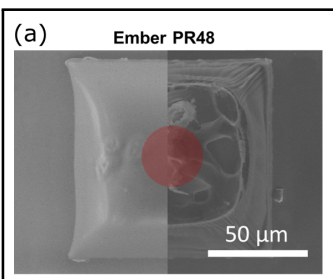

(c) Clean hole $\left(350 \mathrm{~mJ} / \mathrm{cm}^{2}\right)$ :

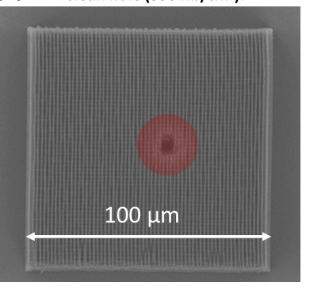

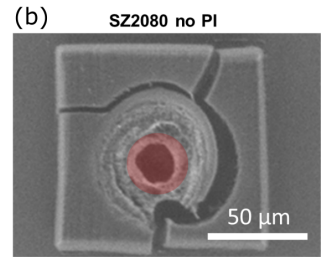

(d) Cracking $\left(850 \mathrm{~mJ} / \mathrm{cm}^{2}\right)$ :

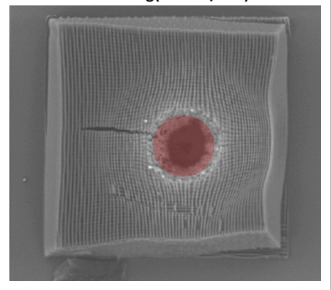

Figure 4. SEM images of optical damage in (a) uniform Ember PR48, (b) uniform SZ2080 without photoinitiator, $(c, d)$ woodpile in SZ2080 without photoinitiator at different incident fluences. 


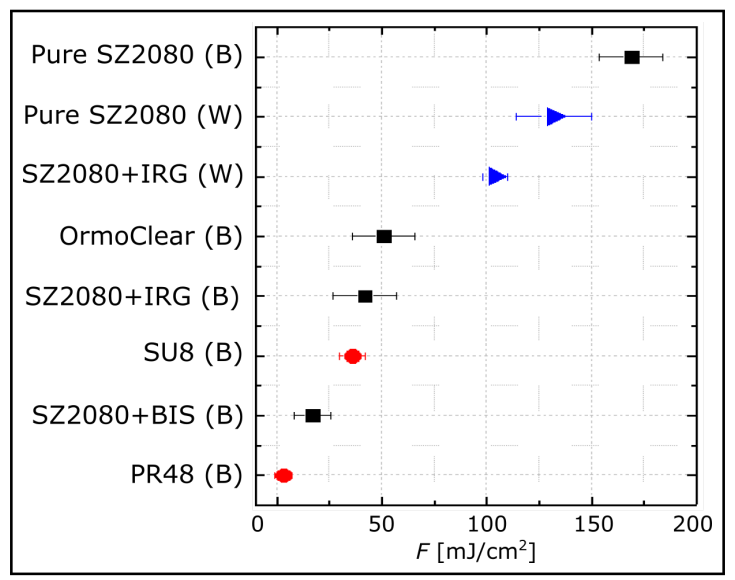

Figure 5. Direct comparison of LIDT between all the tested materials and geometries. Bulk is denoted by letter B and woodpiles by W (these points are also blue). Clear superiority of hybrid polymers (black dots) over organic ones (red dots). The best performing are woodpile and bulk structures made out of pure SZ2080 with latter being the most resilient. 